\title{
The detection of the hidden 'alcoholic' in the accident and emergency department
}

\author{
B. D. HORE \\ Alcoholism Treatment Unit and Detoxification Centre, Withington Hospital, Manchester, \\ England
}

Problems of alcohol misuse are increasingly common and it is estimated that at least $2 \%$ of the population are dependent on alcohol, or drinking at a level which will lead them to develop alcohol-related problems. Further, even for those who are not sufficiently dependent or do not drink consistently enough to cause problems, there are occasions when drinking will lead to difficulties in individuals' lives, such as coming into contact with the law through drunkenness, or becoming injured.

For generations, accident and emergency departments have experienced the problem of drunkenness among patients. In rare instances, accident and emergency departments have access to detoxification centres, where drunken patients can be admitted directly. Thus, during 1983, the Withington Hospital Accident and Emergency Department admitted to the Withington Hospital Detoxification Centre 121 patients.

However, there are only two such purpose-built centres in England and Wales, and generally accident and emergency departments are left to deal with these problems on their own. It is the aim, however, of this article not to discuss the problems created by such patients, nor essentially their management, but to examine ways to improve the detection of those individuals who are alcoholic or alcohol dependent among attenders of accident and emergency departments.

The term alcohol dependence refers to a disorder believed to contain psychological and physical factors, characterized by abnormal patterns of drinking and at its more advanced stage, the presence of a physiological disturbance on withdrawal from alcohol (alcohol withdrawal syndrome). The patterns of abnormal drinking are either the loss of consistency to control alcohol intake and/or inability to abstain. The former refers to that awareness on the part of individuals that when they drink they cannot guarantee to control their drinking on all occasions. On some occasions, control may be possible, as may be abstinence, but the ability to control drinking on all occasions is diminished. The inability to abstain refers to that difficulty to do without alcohol when one wishes to. The alcohol withdrawal syndrome is characterized in its early stages, (that is, 6-8 hours after reducing alcohol intake), by sweating, nausea, retching and tremulousness. The advanced stage of the syndrome is characterized by a confusional state, together

Correspondence: $\operatorname{Dr}$ B. D. Hore, Alcoholism Treatment Unit and Detoxification Centre, Withington Hospital, Nell Lane, West Didsbury, Manchester, M20 8LE, England 
with tremor (delirium tremens). In addition, epileptic seizures may occur at any single $\frac{\mathrm{D}}{\alpha}$ time following withdrawal, but usually within the first 7 days. Rarely, auditory? hallucinations may accompany the visual hallucinations, either as part of the? confusional state, or as an entity in their own right. Some regard this latter condition of auditory hallucinations in clear consciousness as alcoholic hallucinosis, although not allo authorities regard this as due to the withdrawal of alcohol and some consider that a을 similar syndrome can develop when drinking alcohol. The majority of patients, $\frac{\vec{s}}{5}$ however, experience the early form of the syndrome, which naturally occurs on arising 0 in the morning.

It is important to realize that in the early stages of dependence patients present $\overrightarrow{0}$ primarily with problems in areas of social pathology. Thus, in a study of general $\vec{\omega}$ practice, in which general practitioners examined their records of patients who were $\stackrel{\odot}{\circ}$ eventually referred to an alcoholism treatment unit, with a diagnosis of alcoholism 3 . (Hore \& Wilkins, 1976) it was shown that several years before referral, these patientsiv had presented with symptoms of anxiety, depression and gastritis, and much more $\omega$ frequently, with marital problems, difficulty with the law, financial difficulties and work $\stackrel{\vec{\omega}}{\omega}$ problems. It is in the areas of social pathology that patients are likely to present? difficulty to community agencies, such as general practitioners. In relation to accident $\vec{\omega}$ and emergency departments, there are special features characterized by the nature of $\mathbb{D}$ patients attending such departments. However, in such patients alcohol may also be $\stackrel{\oplus}{\oplus}$ causing severe social problems. For example, particularly in city centres, accident and $\bar{\sigma}$ emergency departments usually attract the vagrant alcholic individual. This is a small ${ }^{\infty}$ group of less than $10 \%$ of the total of those who are alcohol dependent, characterized rootlessness and vagrancy. A surprising number (Kessel et al., 1984) have not receivedd. treatment for their dependence. The proportion of those attending a detoxification centre, brought by the police, who were found never to have had previous treatment at a specialized centre for alcoholism was two thirds.

There is also the well-known association between alcohol-dependent individuals and $\frac{\otimes}{\varnothing}$ an increased risk of accidents and violence resulting in injury in individuals who have $\overrightarrow{\vec{O}}$ been drinking at the time. In essence, therefore, the accident and emergency 3 department physician must be alerted to the possibility of alcohol dependence or excessive drinking presenting in a variety of forms. These include:

\section{Drunkenness}

These patients will have to be differentiated from other patients suffering from

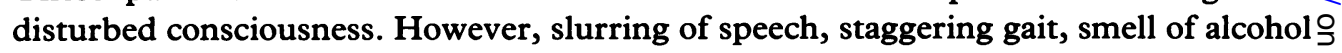
and positive raised alcohol concentration in the blood should not make the diagnosis $\frac{D}{0}$ difficult.

\section{Accidents}

It has been shown clearly that alcohol-dependent individuals have an increased incidence of road traffic, pedestrian, home and industrial accidents. Any patient who presents from whatever background with an accident should, therefore, be regarded as $\stackrel{?}{?}$ potentially suffering from alcohol dependence. 


\section{- Epileptic seizures}

Epileptic seizures occuring as isolated incidents for the first time in middle-aged individuals, have a strong possibility of being due to the effects of alcohol, most frequently with withdrawal. Such seizures may be quite separate from other features of the withdrawal syndrome.

\section{- Trauma}

Patients who have become intoxicated often suffer from trauma. This ranges from minor head injuries to more severe injuries, including sub-dural haematoma and fractured skulls.

\section{- Psychiatric disturbances}

Alcohol-dependent individuals are more likely to kill themselves than those who are not alcohol dependent. Also, in instances of suicide and in attempted suicide, taking alcohol is often part of the suicidal act. In addition, apart from intoxication, many alcoholdependent individuals suffer from depression and anxiety. The former is particularly common in women, who may make strenuous efforts to deny their alcohol problem.

\section{- Alcohol withdrawal syndrome}

This has already been mentioned. Such patients may present, however, with sweating, nausea, retching and tremor, fits or a confusional state.

\section{Alcohol-related diseases}

These include disorders of the nervous system, such as peripheral neuritis and cerebral damage, leading to the Wernicke-Korsakov syndrome and disorders of the hepatic system, including hepatomegaly, due to fatty infiltration, alcohol hepatitis and alcoholic cirrhosis. It is unlikely that many patients will present in this manner to accident and emergency departments.

\section{- Denial}

It has to be remembered at all times that an integral part of the alcohol dependence syndrome is denial of the severity of the drinking problem, which may also extend to denial of the problem by relatives. There is also a considerable ambivalence regarding treatment.

In all these conditions, the accident and emergency physician should be alerted to the possibility that patients, although presenting with other symptoms, have as their basic problem alcohol dependence. Clearly, with intoxicated patients, it is frequently 
impossible to obtain an adequate history and even with those that are not intoxicated, if is, as mentioned below, essential to realize the denial and ambivalence of the individua and relatives. Having been alerted, however, to the possibility of alcohol being the important factor in the patient's condition, the physician must attempt to confirm the् diagnosis of alcohol dependence and also assess the degree of alcohol related problems? The essential information that is required for the former is:

- evidence of one or both of the abnormal patterns of drinking mentioned above;

- evidence of withdrawal symptoms and an assessment of their severity.

Significant withdrawal symptoms indicate an urgent need for treatment and also influence outcome, in that those with more severe withdrawal symptoms are less likeli to do so well. It is therefore important to attract people early into treatment. It must alsङ be remembered that alcohol dependence affects the whole of the population, including one's own medical colleagues. Thus, for example, the cause of a fit in a consultan $\bar{p}$. colleague may well relate to alcohol dependence and no group must be regarded as immune from this condition.

\section{Alcohol-related problems}

Enquiries must also be made about the type of alcohol-related problems most likely to occur. These include difficulties in the marital situation, problems of work, particularlo time off and absenteeism, often of a frequent short nature, trouble with the laww including drunken driving, financial difficulties and of course alcohol-related diseases $s^{\circ}$

It will be seen from the above that the 'front line' of the hospital is particularly likely to receive individuals who have been affected by alcohol. Although the patients mas present with a wide range of problems, the underlying problem relates to their drinking $\frac{5}{9}$ and it is important if this can be diagnosed and the patients referred for appropriate्ष help. There is considerable evidence that treating patients while they still have a degree of social stability and are in the early stages of alcohol dependence is likely to producê better results. The important role linking accident and emergency departments to detoxification centres is also an area that requires further exploration.

\section{REFERENCES}

Hore B. D. \& Wilkins R. H. (1976) A general practice study of the commonest presenting symptoms of alcoholism. Fournal of the Royal College of General Practitioners 26, 140.

Kessel N., Hore B. D., Makenjuola J. D. A., Redmond A. D., Rossall C. J., Rees D. W., Chand T. G.o Gordon M. \& Wallace P. C. (1984) The Manchester Detoxification Service. Lancet i, 839. 